\title{
Chapter 16: Multilevel Analysis of Activity and Actors in Heterogeneous Networked Learning Environments
}

\author{
Daniel D. Suthers \\ Department of Information and Computer Sciences, University of Hawaii, USA \\ DOI: 10.18608/hla17.016
}

\begin{abstract}
Learning in today's networked environments is often distributed across multiple media and sites, and takes place simultaneously via multiple levels of agency and processes. This is a challenge for those wishing to study learning as embedded in social networks, or simply to monitor a networked learning environment for practical purposes. Traces of activity may be fragmented across multiple logs, and the granularity at which events are recorded may not match analytic needs. This chapter describes an analytic framework, Traces, for analyzing participant interaction in one or more digital settings by computationally deriving higher levels of description. The Traces framework includes concepts for modelling interaction in sociotechnical systems, a hierarchy of models with corresponding representations, and computational methods for translating between these levels by transforming representations. Potential applications include identifying sessions of interaction, key actors within sessions, relationships between actors, changes in participation over time, and groups or communities of learners.
\end{abstract}

Keywords: Interaction analysis, social network analysis, community detection, networked learning environments, Traces analytic framework

This chapter is most relevant to readers who will be analyzing trace data of participant interaction in one or more digital settings (which may be of multiple media types), and wish to computationally derive higher levels of description of what is happening, possibly across multiple settings. Examples of these higher levels of description include identifying sessions of interaction, identifying groups or communities of learners across sessions, characterizing interaction in terms of key actors, and identifying relationships between actors. The approach outlined here, the Traces framework, has been used for discovery oriented research, but can also support hypothesis testing research that requires variables at these higher levels of description, or live monitoring of production learning settings using such descriptions. The Traces framework involves a set of concepts for thinking about and modelling interaction in sociotechnical systems, a hierarchy of models with corresponding representations, and computational methods for translating between these levels by transforming representations. These methods have been implemented in experimental software and tested on data from a heterogeneous networked learning environment.

The purpose of this chapter is to introduce the reader to the conceptual and representational aspects of the framework, with brief descriptions of how it can be used for multilevel analysis of activity and actors in networked learning environments. Due to length limitations, detailed examples and information on our implementation and research are not included ${ }^{1}$.

\footnotetext{
${ }^{1}$ See Joseph, Lid, and Suthers (2007) and Suthers (2006) for theoretical background; see Suthers, Dwyer, Medina, and Vatrapu (2010) and Suthers and Rosen (2011) for the development of our analytic representations; see Suthers, Fusco, Schank, Chu, and Schlager (2013) for community detection applications; see Suthers (2015) for an example of how one might combine these capabilities into an activity reporter for monitoring a large networked learning environment. Suthers et al. (2013) and Suthers (2015) describe the data from the Tapped In network of educators we used as a case study in developing this framework. Papers are available at http://lilt.ics. hawaii.edu.
} 


\section{MOTIVATIONS}

Motivations for the Traces framework derive in part from phenomena such as the emergence of Web 2.0 (O'Reilly, 2005) and its adoption by educational practitioners and learners for formal and informal learning, including more recent interest in MOOCs (massive open online courses) (Allen \& Seaman, 2013). In these environments, learning is distributed across time and virtual place (media), and learners may participate in multiple settings. We focus on networked learning environments (NLE), which we define to include any sociotechnical network that involves mediated interaction between participants (hence "networked") in which learning might take place, including for example online communities (Barab, Kling, \& Gray, 2004; Renninger \& Shumar, 2002) and cMOOCs (connectivist MOOCs) (Siemens, 2013). The framework is not applicable to isolated activity by individuals, such as "xMOOCs" in which large numbers of individuals interact primarily with courseware or tutoring systems.

Learning and knowledge creation activities in these networked environments are often distributed across multiple media and sites. As a result, traces of such activity may be fragmented across multiple logs. For example, networked learning environments may include a mashup of threaded discussion, synchronous chats, wikis, microblogging, whiteboards, profiles, and resource sharing. Events may be logged in different formats and locations, disassociating actions that for participants were part of a single unified activity. Integration of multiple sources of trace data into a single transcript may be needed to reassemble data on the interaction. Also, the granularity at which events are recorded may not match analytic needs. For example, media-level events may be the wrong ontology for analyses concerned with relationships between acts, persons, and/or media rather than individual acts. Translation from log file representations to other levels of description may be required to begin the primary analysis.

Derivation of higher levels of description is also motivated by theoretical accounts of learning as a complex and multilevel phenomenon. Theories of how learning takes place in social settings vary regarding the agent of learning, including individual, small group, network, or community; and in the process of learning, including for example information transfer, argumentation, intersubjective meaning-making, shifts in participation and identity, and accretion of cultural capital (Suthers, 2006). Learning takes place simultaneously at all of these levels of agency and with all of these processes, potentially at multiple time scales (Lemke, 2000). A multi-level approach is also motivated by our theoretical stance that social regularities arise from how myriad individual acts are aggregated and influence each other, possibly mediated by artifacts (Latour, 2005), and the methodological implication that to understand phenomena such as actor relationships or community structures, we also need to look at the stream of individual acts out of which these phenomena are constructed. Thus, understanding learning in its full richness requires data that reveal the relationships between individual and collective levels of agency and potentially coordinating multiple theories and methods of analysis (de Laat, 2006; Monge \& Contractor, 2003; Suthers, Lund, Rosé, Teplovs, \& Law, 2013).

\section{TRACES ANALYTIC FRAMEWORK}

This section covers the levels of description and corresponding representations underlying the Traces analytic framework with the next section discussing potential applications. To preview the approach, logs of events are abstracted and merged into a single abstract transcript of events, which is then used to derive a series of representations that support levels of analysis of interaction and of relationships. Three kinds of graphs model interaction. Contingency graphs record how events such as chatting or posting a message are observably related to prior events by temporal and spatial proximity and by content. Uptake graphs aggregate the multiple contingencies between each pair of events to model how each given act may be "taking up" prior acts. Session graphs are abstractions of uptake graphs: they cluster events into spatio-temporal sessions with uptake relationships between sessions. Relationships between actors and artifacts are abstracted from interaction graphs to obtain sociograms and a special kind of affiliation network that we call associograms. The representations used at various levels of analysis are shown schematically in Figure 16.1.

\section{About Transcript}

We begin with various traces of activity (such as log files of events) that provide the source data (Figure 16.1a). These are parsed, using necessarily system-specific methods, into an event stream, as shown in the second level (boxes in Figure 16.1b). Events can come from different media (e.g., chats, threaded discussion, social media), be of various types (e.g., enter chat, exit chat, chat contribution, post message, read message, download file). They should be annotated with time stamps, actors, content (e.g., chat content), and locations (e.g., chat rooms) involved in the event where relevant. The result is an abstract transcript of the distributed activities. By translating from system-specific representations of activity to the abstract transcript, we integrate hitherto fragmented records of activity into 
one analytic artifact.

\section{Contingency Graph}

We then compute contingencies between events (arrows in Figure 16.1b), to produce a model of how acts are mutually contextualized. Human action is contingent upon its setting in diverse ways: computational methods can capture some of the contingencies amenable to automated detection. For example, a contingency called proximal event reflects the likelihood that events occurring close together in time and space are related. In analyzing quasi-synchronous chat, contingencies are installed to prior contributions in the same room that occur within an adjustable time window but not too recently. Address and reply contingencies are installed between an utterance mentioning a user by name and the last contribution and next contribution by that participant within a time window, using a parser/matcher of user IDs to first names. Same actor contingencies are installed to prior acts of a participant over a larger time window to reflect the continuity of an agent's purpose. Overlap in content as represented by sets of lexical stems is used to produce a lexical overlap contingency weighted by the number of overlapping stems. Further contingencies could be computed based on natural language processing methods for analysis of interactional structure (Rosé et al., 2008).
The resulting contingency graph represents the first layer of abstraction (Figure 16.1b), the contextualized action model. In this graph, vertices are events, and contingencies are typed edges between vertices (example types were just described). There may be multiple edges between any two vertices (e.g., two proximal events by the same actor with lexical overlap will have at least three contingencies between them).

\section{Uptake Graph}

It is necessary to collapse the multiple edges between vertices into single edges for two reasons. First, most graph algorithms assume at most only one edge between any two vertices. Second, we are interested in uptake, the relationship between events in which a human action takes up some aspects of prior events as being significant in some manner. Being the fundamental building block of interaction (Suthers et al., 2010), uptake is a basic unit for analysis of how learning takes place in and through interaction. Replying to prior contributions in chats and discussions are examples of uptake, but uptake is not limited to replies: one can appropriate a prior actor's contribution in other ways. Uptake is not specific to a medium: it can occur in different media, and cross media (Suthers et al., 2010). Contingencies are of interest only as collective evidence for uptake, so we abstract the contingency

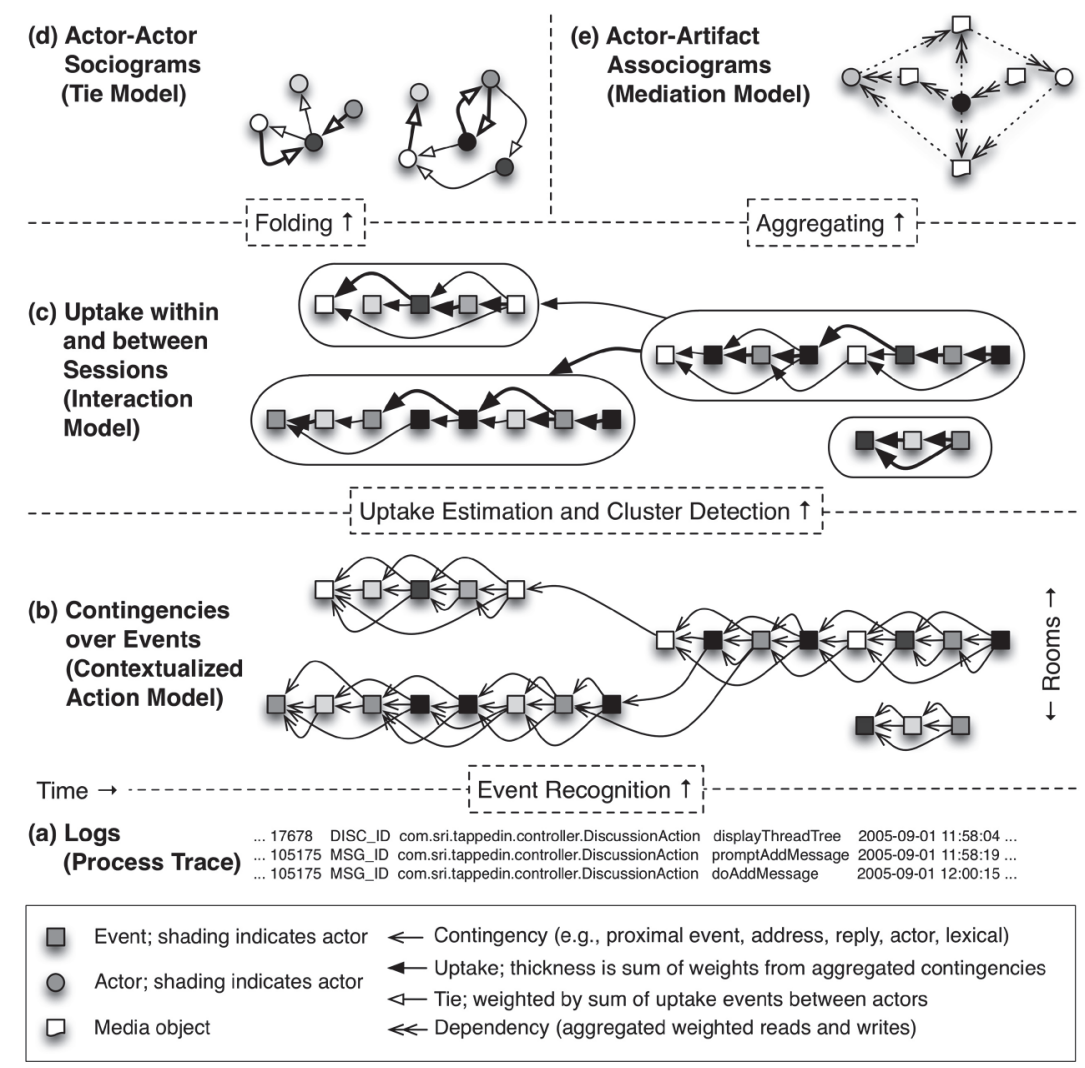

Figure 16.1. Levels of analysis and their representations. 
graph to an uptake graph.

As shown in Figure 16.1c, uptake graphs are similar to contingency graphs in that they also relate events, but they collect together bundles of the various types of contingencies between a given pair of vertices into a single graph edge, weighted by a combination of the strength of evidence in the contingencies and optionally filtering out low-weighted bundles (Suthers, 2015). Different weights can be used for different purposes (e.g., finding sessions, analyzing the interactional structure of sessions, constructing sociograms). Importantly, we do not throw away the contingency weights: these are retained in a vector to summarize the nature of the uptake relation, and, once aggregated into sociograms, of the tie between actors. We can do several interesting things with uptake graphs, but first we usually identify portions of the graph that we want to handle separately, as they represent sessions.

\section{Sessions}

Clusters of events in spatio-temporal proximity are computed to identify sessions (indicated by rounded containers in Figure 16.1c). Methods for doing so are discussed later. For intra-session analysis, the uptake graph for a session is isolated. Several paths are possible from here. For example, the sequential structure of the interaction can be micro-analyzed to understand the development of group accomplishments: this analysis may be difficult to automate. Methods for graph structure analysis can be applied, such as cluster detection, or tracing out thematic threads (Trausan-Matu \& Rebedea, 2010). For inter-session analysis, we collapse each session into a single vertex representing the session, but retain the inter-session uptake links. (For example, there are four sessions in Figure 16.1c and two inter-session uptakes.) These inter-session links indicate potential influences across time and space from one session to another.

\section{Sociograms}

Sociotechnical networks are commonly studied using the methods of social network analysis, using sociogram or sociomatrix representations of the presence or strength of ties between human actors, and graph algorithms that leverage the power of these representations to expose both local (ego-centric) and non-local (network) social structures (Newman, 2010; Wasserman \& Faust, 1994). Either within or across sessions, we can fold uptake graphs into actor-actor sociograms (directed weighted graphs, Figure 16.1d). The tie strength between actors is the sum of the strength of uptake between their contributions. If we want to be stricter about the evidence for relations between the two actors, we may use a different weighting that downplays proximity and emphasizes direct evidence of orientation to the other actor. These sociograms can be analyzed using conventional social network analysis methods, for example centrality metrics to identify key actors.

\section{Associograms}

The sociogram's singular tie between two actors summarizes yet obscures the many interactions between the actors on which the tie is based, as well as the media through which they interacted. To retain the advantages of graph computations on a summary representation while retaining some of the information about how the actors interacted, we use bipartite, multimodal, directed weighted graphs, similar to but more specific than affiliation networks. They are bipartite because all edges go strictly between actors and artifacts and multimodal because the artifact nodes can be categorized into the different kinds of mediators that they represent; for example, chat rooms, discussion forums, and files. Directed edges (arcs) indicate read/write relations or their analogs: an arc goes from an actor to an artifact if the actor has read that artifact (e.g., opened a discussion message or was present when someone chatted), and from an artifact to an actor if the actor modified the artifact (e.g., posted a discussion message or chatted). The direction of the arc indicates a form of dependency, the reverse direction of information flow. Weights on the arcs indicate the number of events that took place between the corresponding actor/artifact pair in the indicated direction. Since "affiliation network" is not specific enough and "bipartite multimodal directed weighted graph" is too long, to highlight their unique nature we call these graphs associograms (Suthers \& Rosen, 2011). This term is inspired by Latour's (2005) concept that social phenomena emerge from dynamic networks of associations between human and non-human actors.

For example, Figure 16.2 shows a portion of an associogram from the Tapped In educator network,

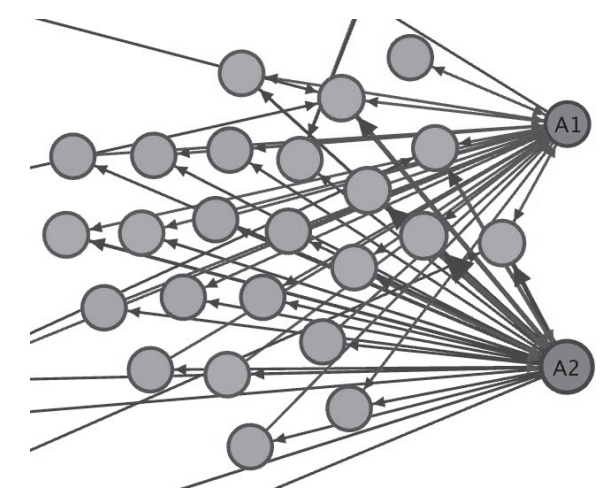

Figure 16.2. An associogram from the Tapped In data. Actors represented by nodes on the right have read and written to the files and discussions represented by differently coloured nodes on the left. 
representing asymmetric interaction between two actors, with one actor writing most of the files and another writing to most of the discussions. A sociogram consisting of a single link between these two actors would fail to capture this information. The associogram retains information about the distribution of activity across media. Network analytic methods can then simultaneously tell us how both human actors and artifacts participate in generating the larger phenomena of interest, such as the presence of communities of actors and the media through which they are technologically embedded (Licoppe \& Smoreda, 2005). Although interaction is not directly represented, the associogram also provides a bridge to the interaction level of analysis (Suthers et al., 2010), allowing us to retrieve activity in specific media settings.

\section{EXAMPLES OF ANALYTIC OPTIONS}

The Traces framework provides multiple pathways for analysis. In the following sections we illustrate various analyses that can be supported by this framework (Suthers \& Dwyer, 2015). These examples are from analyses we have done with our experimental software implementation.

\section{Identifying Sessions of Interaction}

Different options exist for detection of sessions in interaction graphs. If interaction is not clearly demarcated by periods of non-interaction and one wishes to discover clusters of high activity, we have found that cohesive subgraph detection or "community detection" algorithms (Fortunato, 2010) such as modularity partitioning (Blondel, Guillaume, Lambiotte, \& Lefebvre, 2008) applied to uptake graphs are useful (Suthers, 2017). If (as in our Tapped In data) activity is distributed across rooms and the activity within a room almost always has periods of non-activity between sessions, sessions can be identified efficiently without needing to

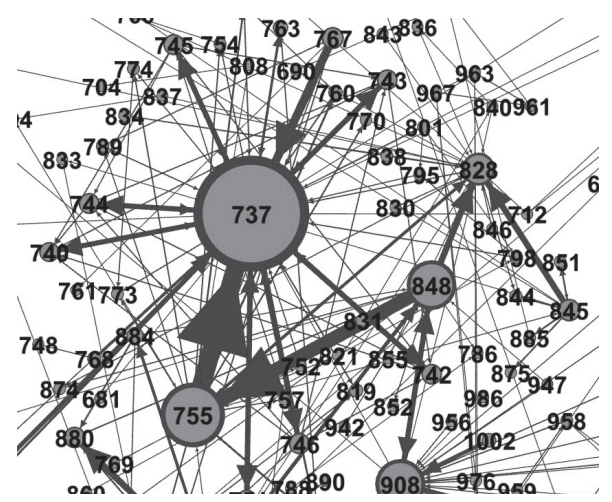

Figure 16.3. Close-up of session graph with inter-session uptake. Node and edge size is weighted degree. construct a contingency graph (it can be constructed later for other purposes). Activity is tracked in each room, and a new session ID is assigned to the room every time there is a gap of $\mathrm{S}$ seconds of no activity. $\mathrm{S}$ is a tunable parameter, such as 240 seconds. Suthers (2017) discusses these options further.

\section{Tracing Influences Between Sessions}

We might be interested in non-local influences between sessions across time and space. Uptake relations between events in different sessions can be aggregated into weighted uptake relations between sessions (Figure 16.1c). An example session graph from Suthers (2015) is shown in Figure 16.3. Some sessions have more heavily weighted links between them. Reading the edges in reverse order (uptake points backwards in time), we see that session 737 (in the Reception room) influenced session 755 (Teaching Teachers room), which in turn influenced 848 (NTraining room). Examination of the rooms and participants involved showed that many participants logged into or met in the Reception room, then went to Teaching Teachers for session 755 on mentoring in the schools. Then the facilitator of 755 announced that she had another session on teacher training in another room: several participants in the mentoring session followed her to NTraining for session 848. Further details are in Suthers (2015).

\section{Identifying Actor Roles and Tracking Change in Participation Over Time}

Educators or NLE facilitators may want to identify the key participants in their online learning communities, whether for assessment in formal educational settings, to encourage volunteers in participant-driven settings, or for research purposes such as to study what drives key participants. It is also important to know who is disengaged. Some of these needs can be met through social network analysis. We can generate sociograms for any granularity of the uptake graph (e.g., within a session, or across sessions over a time period) by folding uptake relations between events into ties between their actors. For example, a facilitator might want to see a sociogram summarizing actor activity in session 755, the session on mentoring teachers led by MT. The sociogram is shown in Figure 16.4. Node size is weighted in-degree, discussed below.

Sociograms add information over mere counts of number of contributions because some sociometrics are sensitive to the network context of nodes representing actors. For example, weighted in-degree indicates the extent to which other acts have contingencies to and hence potentially took up a given act. Aggregating these acts for an actor is an estimate of how much an actor's contributions are taken up by others. This metric is sensitive to both the level of activity of the actor and that activity's relation to others' activity. Weighted 
out-degree is an estimate of how much an actor takes up others' contributions. Eigenvector centrality (and its variants such as PageRank and Hubs and Authorities) is a non-local metric that takes into account the centrality of one's neighbours (Newman, 2010, p. 169), indicating the extent to which an actor is connected to others who are themselves central. Betweeness centrality is an indicator of actors who potentially play brokerage roles in the network: high betweeness centrality means that the node representing an actor is on relatively more shortest paths between other actors (Newman, 2010, p. 185), so potentially controls information flow or mediates contact between these actors. Betweeness is of particular interest when examining activity across sessions: different sessions generally have different actors, so an actor attending multiple sessions will have high betweeness.

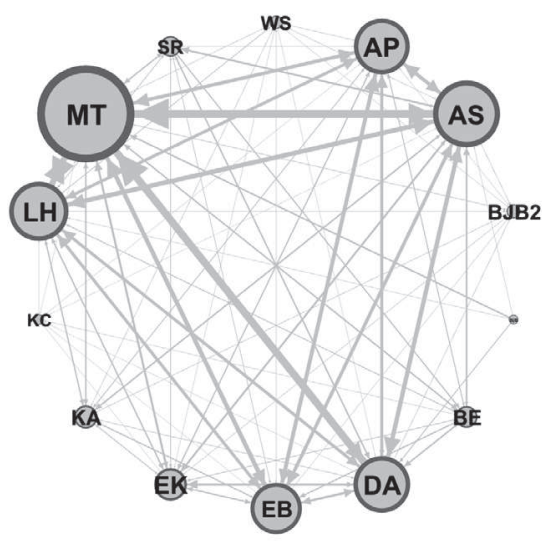

Figure 16.4. Sociogram from a Tapped In session showing prominent actors and their interactions.

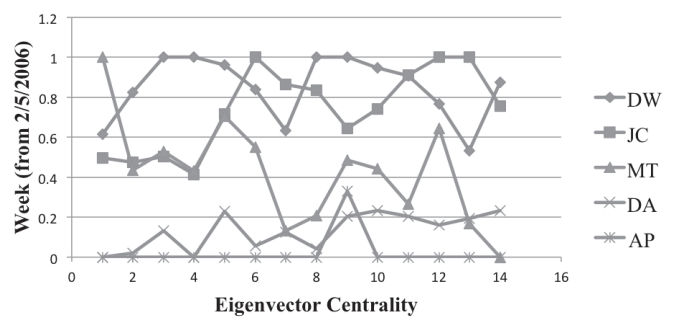

Figure 16.5. Eigenvector Centrality of several actors in Tapped In over a 14 week period.

Analyses on longer time scales may be of interest to researchers as well as practicing educators. One can trace the development of actors' roles over time in terms of changes in their sociometrics. For example, one might aggregate uptake for all actors in the network into sociograms at one week intervals, and then graph the sociometrics on a weekly basis, looking for trends. One can see some of these trends in Figure 16.5, taken from Suthers (2015). The plots for sustaining actors will remain high (e.g., DW, a volunteer guide), and for those who return for periodic events will exhibit a regular spiking structure (e.g., MT, who facilitated monthly events). Steadily increasing or decreasing metrics indicate persons becoming more or less engaged, respectively (possibly DA), and single spikes indicate a one-off visit (AP).

Superficially, these analyses appear similar to the many sociometric analyses found in the literature, so we should highlight what the Traces framework has added. Our implementation of the Traces framework derived these latent ties from automated interaction analysis of streams of events, by identifying and then aggregating multiple contingencies between events, and then folding the resulting uptake relations between events into an actor-actor graph. This has significant advantages over, for example, manual content analysis or the use of surveys to derive tie data, which are labour intensive, or reliance on explicit "friending" relations: surveys and friend links may not reflect the latent ties in actual interaction between the persons in question. Another advantage is described below.

\section{Identifying Relationships Between Actors}

The Traces framework derives ties between actors by aggregating multiple contingencies between their contributions. The contingencies indicate the qualitative nature of the relationship between these contributions, e.g., being close in time and space, using the same words, and addressing another actor by name. When contingencies are aggregated into uptake relations, we keep track of what each type of contingency contributed to the uptake relation. This record keeping is continued when folding uptakes into ties, so that for any given pair of actors we have a vector of weights that provides information about the nature of the relationship in terms of the underlying contingencies. For example, we can quantify how the relationship between DA and MT was enacted over a given time period in terms of how often they chatted in proximity to each other, the lexical overlap of their chat contents, and how often they addressed each other by name in each direction. Relational information might be of interest to educators or researchers who are managing collaborative learning activities amongst students, or even to examine one's own relations to students. The Traces framework makes this possible by retaining information about the interactional origins of ties (see Suthers, 2015).

\section{Identifying Groups or Communities of Learners Across Sessions}

In the network analysis literature, "community detection" refers to finding subgraphs of mutually associated vertices under graph-theoretic definitions, rather than to sociological concepts of community (e.g., Cohen, 1985; Tönnies, 2001). However, we can use the former 
as evidence for the latter, particularly when studying networked societies (Castells, 2001; Wellman et al., 2003). A good graph-theoretic definition should capture the intuition that individuals in a sociological community are more closely associated with each other than they are with individuals outside of their community. Algorithms based on the modularity metric are widely used in the literature for this purpose. The modularity metric (Newman, 2010, p. 224) compares the density of weighted links inside (non-overlapping) partitions of vertices to weighted links expected in a random graph, to find highly modular partitions. Finding the best possible partition under a modularity metric is computationally impractical on large networks, but a fast algorithm known as the Louvain method (Blondel et al., 2008) has been shown to give good approximations. An example is shown in Figure 16.6.

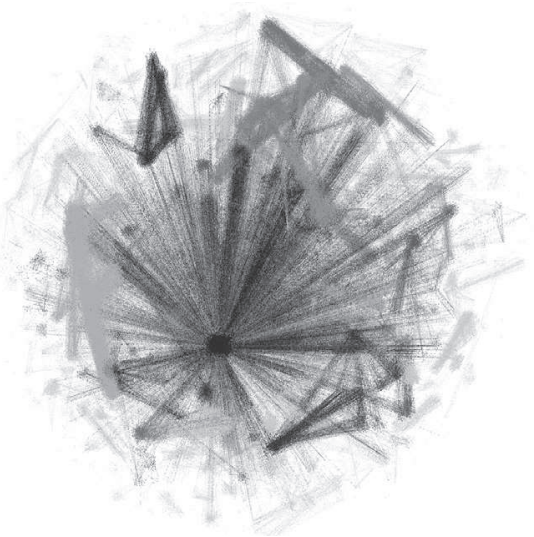

Figure 16.6. OpenOrd visualization of partitions found in combined associogram for actors associated via chats, discussions, and files in Tapped In.

Once partitions have been obtained, one can characterize the community structure of the network in several ways. Quantitative summaries can include the distribution of partition size (e.g., is there primarily one large community, or does the network contain many small and a few large communities?) and distributions of parameters across sizes (e.g., how does activity per actor relate to community size, and how does the use of different media vary with community size?). Qualitative characterization of community structure requires examining the attributes of actors and the media through which they interact to interpret each partition. See Suthers, Fusco, et al. (2013) for examples of both quantitative and qualitative analyses of the partitioning shown in Figure 16.6.

\section{SUMMARY AND RELATED WORK}

This chapter introduced the Traces analytic framework, which integrates traces of activity distributed across media, places, and time into an abstract transcript, and then provides a linked abstraction hierarchy using observable contingencies between events to build models of interaction and ties. Contingencies are applied to events in the abstract transcript to produce a contingency graph. Contingencies are then aggregated into uptake between the same events. Uptake that crosses partitions can be used to identify influences across space and time, and uptake within partitions can be analyzed to study the interactional structure of a session. Uptake graphs can be folded into networks where nodes are actors rather than events, to which sociometrics are applied. Events can also be folded into actor-artifact networks or "associograms" that capture how actors are associated with each other via mutual read and write of media objects. The framework addresses the need to understand how aggregate phenomena (e.g., "ties," "roles," and "communities") are both produced by and provide the setting of specific interactional events. It has been implemented as experimental software and tested with data from a heterogeneous networked learning community.

Other authors have noted the need to combine multiple forms of analysis, including specifically social network analysis in networked learning environments. For example, de Laat, Lally, Lipponen, and Simons (2007) and Martínez and colleagues (2006) showed the utility of combining social network analysis with various qualitative and quantitative methods in the study of participation networks. Others have constructed and folded interaction graphs into sociograms of ties between actors. For example, Rosen and Corbit (2009) constructed graphs based on temporal proximity, and Haythornthwaite and Gruzd (2008) describe preliminary work in extracting interaction relations from references and names. The Traces framework is in the same spirit, but is arguably more mature. We consider multiple kinds of relations between events to provide a richer basis for session identification and subsequent analysis of activity and actors within sessions, and have automated these analyses and tested them on a rich historical data corpus where diverse participants interacted in an environment exhibiting many features of today's distributed interaction. Work by Trausan-Matu on "polyphonic analysis" (Trausan-Matu \& Rebedea, 2010) has affinities to our use of multiple contingencies, but has only recently been abstracted to higher levels of analysis. A thesis by Charles (2013) has provided an alternative implementation of our approach and extended the set of contingencies. Our approach dovetails with work that applies natural language processing methods for analysis of interactional structure, and indeed rules for generating additional contingencies could be derived from such research. Although our software is 
not presently in a form suitable for distribution, the author hopes that the Traces framework can serve the reader as a way to organize their own analyses of heterogeneous networked learning environments and other sociotechnical systems.

\section{ACKNOWLEDGMENTS}

Nathan Dwyer, Kar-Hai Chu, Richard Medina, Devan Rosen, and Ravi Vatrapu co-developed the ideas behind this work. Nathan Dwyer and Kar-Hai Chu also wrote the core software implementation. Mark Schlager, Patti Schank, and Judi Fusco shared data and expertise. This work was partially supported by NSF Award 0943147.

\section{REFERENCES}

Allen, I. E., \& Seaman, J. (2013). Changing course: Ten years of tracking online education in the United States. Babson Survey Research Group. http://www.onlinelearningsurvey.com/reports/changingcourse.pdf

Barab, S. A., Kling, R., \& Gray, J. H. (2004). Designing for virtual communities in the service of learning. New York: Cambridge University Press.

Blondel, V. D., Guillaume, J.-L., Lambiotte, R., \& Lefebvre, E. (2008). Fast unfolding of communities in large networks. Journal of Statistical Mechanics: Theory and Experiment. doi:10.1088/1742-5468/2008/10/P10008

Castells, M. (2001). The Internet galaxy: Reflections on the Internet, business, and society. Oxford, UK: Oxford University Press.

Charles, C. (2013). Analysis of communication flow in online chats. Unpublished Master's Thesis, University of Duisburg-Essen, Duisburg, Germany. (2245897)

Cohen, A. P. (1985). The symbolic construction of community. New York: Routledge.

de Laat, M. (2006). Networked learning. Apeldoorn, Netherlands: Politie Academie.

de Laat, M., Lally, V., Lipponen, L., \& Simons, R.-J. (2007). Investigating patterns of interaction in networked learning and computer-supported collaborative learning: A role for social network analysis. International Journal of Computer Supported Collaborative Learning, 2(1), 87-103.

Fortunato, S. (2010). Community detection in graphs. Physics Reports, 486, 75-174.

Haythornthwaite, C., \& Gruzd, A. (2008). Analyzing networked learning texts. In V. Hodgson, C. Jones, T. Kargidis, D. McConnell, S. Retalis, D. Stamatis, \& M. Zenios (Eds.), Proceedings of the $6^{\text {th }}$ International Conference on Networked Learning (NLC 2008), 5-6 May 2008, Halkidiki, Greece (pp. 136-143).

Joseph, S., Lid, V., \& Suthers, D. D. (2007). Transcendent communities. In C. Chinn, G. Erkens, \& S. Puntambekar (Eds.), Proceedings of the $7^{\text {th }}$ International Conference on Computer-Supported Collaborative Learning (CSCL 2007), 16-21 July 2007, New Brunswick, NJ, USA (pp. 317-319). International Society of the Learning Sciences.

Latour, B. (2005). Reassembling the social: An introduction to actor-network-theory. New York: Oxford University Press.

Lemke, J. L. (2000). Across the scales of time: Artifacts, activities, and meanings in ecosocial systems. Mind, Culture E Activity, 7(4), 273-290.

Licoppe, C., \& Smoreda, Z. (2005). Are social networks technologically embedded? How networks are changing today with changes in communication technology. Social Networks, 27(4), 317-335.

Martínez, A., Dimitriadis, Y., Gómez-Sánchez, E., Rubia-Avi, B., Jorrín-Abellán, I., \& Marcos, J. A. (2006). Studying participation networks in collaboration using mixed methods. International Journal of Computer-Supported Collaborative Learning, 1(3), 383-408.

Monge, P. R., \& Contractor, N. S. (2003). Theories of communication networks. Oxford, UK: Oxford University Press.

Newman, M. E. J. (2010). Networks: An introduction. Oxford, UK: Oxford University Press. 
O'Reilly, T. (2005). What is Web 2.0: Design patterns and business models for the next generation of software. http://www.oreillynet.com/pub/a/oreilly/tim/news/2005/09/30/what-is-web-20.html

Renninger, K. A., \& Shumar, W. (2002). Building virtual communities: Learning and change in cyberspace. Cambridge, UK: Cambridge University Press.

Rosé, C. P., Wang, Y.-C., Cui, Y., Arguello, J., Stegmann, K., Weinberger, A., \& Fischer, F. (2008). Analyzing collaborative learning processes automatically: Exploiting the advances of computational linguistics in computer-supported collaborative learning. International Journal of Computer-Supported Collaborative Learning, 3(3), 237-271. doi:10.1007/s11412-007-9034-0

Rosen, D., \& Corbit, M. (2009). Social network analysis in virtual environments. Proceedings of the $20^{\text {th }} \mathrm{ACM}$ conference on Hypertext and Hypermedia (HT '09), 29 June-1 July 2009, Torino, Italy (pp. 317-322). New York: ACM.

Siemens, G. (2013). Massive open online courses: Innovation in education? In R. McGreal, W. Kinuthia, S. Marshall, \& T. McNamara (Eds.), Open educational resources: Innovation, research and practice (pp. 5-15). Vancouver, BC: Commonwealth of Learning and Athabasca University.

Suthers, D. D. (2006). Technology affordances for intersubjective meaning-making: A research agenda for CSCL. International Journal of Computer-Supported Collaborative Learning, 1(3), 315-337.

Suthers, D. D. (2015). From micro-contingencies to network-level phenomena: Multilevel analysis of activity and actors in heterogeneous networked learning environments. Proceedings of the $5^{\text {th }}$ International Conference on Learning Analytics and Knowledge (LAK 'LA), 16-20 March, Poughkeepsie, NY, USA (pp. 368-377). New York: ACM.

Suthers, D. D. (2017). Applications of cohesive subgraph detection algorithms to analyzing socio-technical networks. Proceedings of the 50 ${ }^{\text {th }}$ Hawaii International Conference on System Sciences (HICSS-50), $4-7$ January 2017, Waikoloa Village, HI, USA (CD-ROM). IEEE Computer Society.

Suthers, D. D., \& Dwyer, N. (2015). Identifying uptake, sessions, and key actors in a socio-technical network. Proceedings of the $44^{\text {th }}$ Hawaii International Conference on System Sciences (HICSS-44), 5-8 January 2011, Kauai, Hawai'i (CD-ROM). IEEE Computer Society.

Suthers, D. D., Dwyer, N., Medina, R., \& Vatrapu, R. (2010). A framework for conceptualizing, representing, and analyzing distributed interaction. International Journal of Computer Supported Collaborative Learning, 5(1), 5-42. doi:10.1007/s11412-009-9081-9

Suthers, D. D., Fusco, J., Schank, P., Chu, K.-H., \& Schlager, M. (2013). Discovery of community structures in a heterogeneous professional online network. Proceedings of the $46^{\text {th }}$ Hawaii International Conference on the System Sciences (HICSS-46), 7-10 January 2013, Maui, HI, USA (CD-ROM). IEEE Computer Society.

Suthers, D. D., Lund, K., Rosé, C. P., Teplovs, C., \& Law, N. (2013). Productive multivocality in the analysis of group interactions. Springer.

Suthers, D. D., \& Rosen, D. (2011). A unified framework for multi-level analysis of distributed learning. In G. Conole, D. Gašević, P. Long, \& G. Siemens (Eds.), Proceedings of the $1^{\text {st }}$ International Conference on Learning Analytics and Knowledge (LAK 'LA), 27 February-1 March 2011, Banff, AB, Canada (pp. 64-74). New York: ACM.

Tönnies, F. (2001). Community and civil society (J. Harris \& M. Hollis, Trans. from Gemeinschaft und Gesellschaft, 1887) Cambridge, UK: Cambridge University Press.

Trausan-Matu, S., \& Rebedea, T. (2010). A polyphonic model and system for inter-animation analysis in chat conversations with multiple participants. In A. Gelbukh (Ed.), Computational linguistics and intelligent text processing (pp. 354-363). Springer.

Wasserman, S., \& Faust, K. (1994). Social network analysis: Methods and applications. New York: Cambridge University Press.

Wellman, B., Quan-Haase, A., Boase, J., Chen, W., Hampton, K., Diaz, I., \& Miyata, K. (2003). The social affordances of the Internet for networked individualism. Journal of Computer-Mediated Communication, 8(3). doi:10.1111/j.1083-6101.2003.tb00216.x 